\title{
Direct Solid-State Fermentation of Soybean Processing Residues for the Production of Fungal Chitosan by Mucor rouxii
}

\author{
Andro Mondala*, Ramea Al-Mubarak, James Atkinson, Shaun Shields, Brian Young, \\ Yurguen Dos Santos Senger, Jan Pekarovic \\ Department of Chemical and Paper Engineering, Western Michigan University, Kalamazoo, USA \\ Email: ${ }^{*}$ andro.mondala@wmich.edu
}

Received 8 January 2015; accepted 27 January 2015; published 4 February 2015

Copyright (C) 2015 by authors and Scientific Research Publishing Inc.

This work is licensed under the Creative Commons Attribution International License (CC BY).

http://creativecommons.org/licenses/by/4.0/

(c) (i) Open Access

\begin{abstract}
The feasibility of utilizing soybean-processing residues such as soybean meal and hulls as substrates for chitosan production by the fungus Mucor rouxii ATCC 24905 via solid-state fermentation (SSF) was investigated. The effects of the type of soybean-based substrate, length of cultivation period, substrate moisture content, substrate $\mathrm{pH}$, incubation temperature and extraction conditions on chitosan yield were determined. The results showed that a maximum fungal chitosan yield of up to $3.44 \%$ by dry substrate weight $(34.4 \mathrm{~g} / \mathrm{kg})$ could be achieved using a pure soybean meal substrate with an initial moisture content of $50 \%(\mathrm{w} / \mathrm{w})$ and $\mathrm{pH}$ of 5 - 6 incubated for six days at $25^{\circ} \mathrm{C}$. A more severe heat treatment (autoclaving vs. refluxing) resulted in higher chitosan extraction yields regardless of the strength of extraction reagents. Fourier transform infrared (FTIR) analysis of the fungal chitosan revealed its degree of deacetylation (DDA) to be between $55 \%$ and $60 \%$.
\end{abstract}

\section{Keywords}

Solid-State Fermentation, Fungal Chitosan, Biopolymers, Bioconversion, Agro-Industrial Residues

\section{Introduction}

Chitosan is a natural biopolymer that has gained increased attention in recent years due to its interesting and beneficial biological and molecular characteristics, which enable its application in a wide variety of industrial and biotechnological fields. These fields range from agriculture, environmental remediation, pulp and paper

${ }^{*}$ Corresponding author.

How to cite this paper: Mondala, A., Al-Mubarak, R., Atkinson, J., Shields, S., Young, B., Senger, Y.D.S. and Pekarovic, J. (2015) Direct Solid-State Fermentation of Soybean Processing Residues for the Production of Fungal Chitosan by Mucor rouxii. Journal of Materials Science and Chemical Engineering, 3, 11-21. http://dx.doi.org/10.4236/msce.2015.32003 
coatings and barrier films, food preservation, and cosmetics to more advanced technology emerging fields such as functional materials in flexible printed organic electronic devices, protein separations, drug delivery, and tissue engineering [1]. Chitosan is a heterogeneous polysaccharide containing $\beta$-(1-4)-linked 2-amino-2-deoxy-Dglucose (glucosamine) and $\mathrm{N}$-acetyl-2-amino-2-deoxy-D-glucose ( $\mathrm{N}$-acetyl glucosamine) units, with the former consisting of more than $60 \%$ of the copolymer [2]. It is obtained through deacetylation of chitin, which contains mostly $N$-acetyl glucosamine units. Its unique and beneficial properties enabling its numerous applications include its cationic nature, chelation and ion-binding ability, protein immobilization, film- and gel-forming characteristics, chemical reactivity, amenability to modification, biocompatibility, and antimicrobial activity [3]. These properties are a consequence of its heterogeneous molecular structure and the presence of highly reactive amine groups at the glucosamine residues [4]. Fields and applications that can be served by utilizing chitosan and exploiting its properties include biomedical engineering, food and agriculture, environmental remediation, and polymers, films, fibers and coatings [1]-[3].

Chitosan is traditionally produced by subjecting crustacean shells to severe alkali, acid, and/or enzymatic treatments to isolate chitin, by removing impurities such as proteins, minerals and pigments, and by deacetylating of chitin to chitosan - that is, the removal of the acetyl groups in the $\mathrm{N}$-acetyl glucosamine units comprising the majority of chitin to increase the proportion of glucosamine units in the polymer. Harsh reaction conditions are required due to the complex organization of chitin with other components in the crustacean exoskeleton [5]. Thus, the conventional process results in high labor and processing costs. Additionally, these raw materials are seasonally and geographically limited and have highly variable characteristics, which can lead to inconsistent product yield and molecular characteristics. This is undesirable for high purity applications such as in the pharmaceutical and biomedical fields [3] [6]. On the other hand, chitosan is also found as a major fungal cell wall component particularly of fungal strains belonging to the order Mucorales of the Zygomycetes class [6] [7]. These fungal species produce chitosan directly unlike crustacean-derived chitosan, which has to be produced via isolation and chemical conversion of chitin from the exoskeletons of these organisms. Fungal chitosan is more advantageous than crustacean-based chitosan, because the former can be produced in a controlled environment all year round independent of the seasonal seafood industry [8]. Fungal growth and chitosan productivity and properties can be controlled through the manipulation of fermentation and extraction conditions to give chitosan more consistent and desired physico-chemical properties compared with chitosan obtained from crustacean sources [9]. Moreover, the extraction process for isolating chitosan from fungal cell walls is relatively simpler and milder than obtaining chitosan from crustacean shells, thus producing less waste [9]. In terms of chitosan properties, chitosan produced by most of the identified producer fungal strains has roughly the same degree of deacetylation, and relatively lower viscosities and molecular weight than crustacean-derived chitosan, which improves its potential for processing and modification for its numerous applications particularly in agriculture and biomedical engineering [10].

Agro-industrial crops, by-products and residues can be used as inexpensive carbon sources for growing the fungi, which can help alleviate some environmental concerns on the disposal of these waste materials [11]. These materials are particularly suited for use as substrates and microbial growth support in solid-state fermentation (SSF) processes, which exploit the natural growth conditions of filamentous fungi and their capabilities to biosynthesize and excrete hydrolytic enzymes to directly ferment solid biomass substrates. SSF methods have been applied previously for the production of fungal chitosan using a variety of agro-industrial residues and fungal strains, though not as prevalent as using submerged liquid fermentation (SLF) cultures. Potato chip processing wastes [12], cottonseed hulls and corn residues [13], sweet potato [14], and residues from the manufacture of soymilk and mungbean noodles [15] were utilized as fermentation substrates for growth and chitosan production of various filamentous fungal strains. The filamentous fungus Mucor rouxii, a Zygomycete strain, has been well known to produce high amounts of chitosan using SLF [8] [16] [17]. The possibility of utilizing this fungus for SSF of agro-residues for chitosan production has not been tested previously.

Soybean processing residues, such as soybean meal and soy hulls, are excellent options for use as fermentation media/substrates for the growth of fungi that can produce chitosan due to their abundance and high nutrient content. According to the American Soybean Association, one bushel $(60 \mathrm{lbs})$ of soybeans contain on a mass basis approximately $18.3 \%$ oil, $80 \%$ meal, and $1.7 \%$ of other residues such as the hulls and mill runs [18].With a total annual soybean production of 89.5 million metric tons, the amount of soymeal production is approximately 71.6 million metric tons while that of soybean hulls is estimated to be 1.5 million metric tons [19]. The conventional use of soybean meal includes supplementation of animal feed due to its high carbohydrate and protein 
content. However, such use is limited due to the presence of oligosaccharides that are indigestible by non-ruminant livestock and poultry [20]. To some extent, soy hulls can be used as animal feed fillers. But due to their relatively low protein and high fiber content, they are not as widely used as soymeal for this application. Instead, they are used as a boiler fuel additive. These characteristics, however, can make both soybean meal and hulls an ideal solid-state fermentation (SSF) substrate for the growth and cultivation of fungi that can be used to produce chitosan. The fungal chitosan product, with all its interesting molecular properties and applications, can be used as a renewable and "green" biobased alternative not only for crustacean chitosan, but also for petroleum based polymers.

The goal of this study is to investigate fungal chitosan production via solid-state fermentation of soybean processing residues using Mucor rouxii. Experiments were conducted to determine the optimum cultivation and extraction conditions for maximum chitosan yield. The physicochemical characteristics of the produced chitosan were then determined using analytical methods.

\section{Experimental}

\subsection{Materials}

Mucor (Amylomyces) rouxii ATCC 24905, the fungal strain used in this study, was purchased from the American Type Culture Collection (ATCC). The freeze-dried culture was rehydrated with sterile deionized (DI) water and stock cultures were generated by cultivation of the rehydrated fungal cells on potato dextrose agar (PDA) slants at $25^{\circ} \mathrm{C}$ for five days. The stock cultures were then stored and maintained at $4^{\circ} \mathrm{C}$ until use. Soybean meal and hulls provided by three local soybean processors was tested as a solid-state fermentation substrate. These were then stored in airtight plastic containers in a refrigerator at $4^{\circ} \mathrm{C}$ until use. Representative samples of the soybean meal and hulls from the different sources were sent to Northland Labs, Inc. (Northbrook, IL) for analysis of carbohydrates, proteins, moisture, and ash using standard AOAC methods. The soymeal were analyzed and used as received while whole soy hulls were homogenized to granular form using a Waring blender. Composite samples consisting of equal amounts from each of the three suppliers were used for the fermentation process. Commercial grade sodium hydroxide pellets and Optima grade glacial acetic acid were purchased from Fisher Scientific (Pittsburgh, PA, USA). Low molecular weight chitosan purchased from Sigma Aldrich (St. Louis, MO, USA) was used as the reference chitosan for fungal chitosan characterization. The chemicals and standards were used as received.

\subsection{Fungal SSF Cultivation and Chitosan Production}

The fungal spore suspension inocula used in the SSF cultures were prepared as follows: ten milliliters of sterile DI water was transferred aseptically into a $M$. rouxii ATCC 24905 stock PDA slant. The slant tube was then gently inverted ten times to suspend the fungal spores in water. After this, 2-mL of the spore suspension was immediately transferred aseptically into a new PDA plate. The spore suspension was distributed onto the PDA surface using a sterile disposable L-spreader. The inoculated plates were incubated at $30^{\circ} \mathrm{C}$ for five days. After the incubation period, the entire mycelial growth on the agar surface was scraped using a sterile metal spatula and transferred into a $250-\mathrm{mL}$ flask containing $100 \mathrm{~mL}$ of sterile DI water. The mixture was gently shaken to suspend the fungal spores in the water. After this, the suspension was filtered through a sterile coarse filter paper into a new sterile collecting flask to remove the large agar and fungal debris.

The soybean residue substrates were prepared and inoculated as a single batch in a sterile autoclavable plastic bag. Sterile DI water was added to the filtered fungal spore suspension to attain the desired initial moisture content setting $(30 \%, 50 \%$, or $70 \% \mathrm{w} / \mathrm{w})$. The $\mathrm{pH}$ of the sterile culture dilution water was previously set according to the desired initial $\mathrm{pH}$ setting of the substrate $(\mathrm{pH} 4,5$, or 6$)$. The wet soymeal mash was shaken and mixed thoroughly inside the bag and then distributed in 10-gram portions (wet basis) in sterile polystyrene deep petri dishes. The petri dishes containing the soybean meal fungal cultures were then incubated at the desired temperature setting $\left(25^{\circ} \mathrm{C}, 30^{\circ} \mathrm{C}\right.$, or $\left.35^{\circ} \mathrm{C}\right)$ for the set cultivation period $(3,6,9,12$, or 15 days). All experimental treatments were tested in triplicate petri dish cultures.

\subsection{Measurement of Fungal Growth}

The extent of growth of $M$. rouxii in the soybean meal cultures was determined indirectly by spectrophotometric 
measurement of glucosamine in samples of the fermented soymeal substrate according to a method published elsewhere [21].

\subsection{Chitosan Extraction}

Fungal chitosan produced in the soybean residue cultures was extracted and isolated using an approach based on previously published methods [12] [22] [23]. The entire contents of each petri dish soy residues culture were processed first by extraction of residual proteins and other alkali soluble materials with $\mathrm{NaOH}$ solution with the desired concentration ( $1 \mathrm{M}$ or $46 \% \mathrm{w} / \mathrm{v})$ and volume-to-solids ratio (30:1 or 10:1, mL/g dry mass). Two different heated extraction methods were also tested: autoclaving at $121^{\circ} \mathrm{C}, 15$ psi for 15 min or refluxing at $46^{\circ} \mathrm{C}$ for $8 \mathrm{~h}$. Alkali insoluble materials (AIMs) were recovered by vacuum filtration and washed to neutral pH with DI water. The washed AIMs were transferred and weighed into tared glass petri dishes and dried in an oven at $60^{\circ} \mathrm{C}$ overnight or until a constant weight was achieved. The dry weight and moisture content of the AIMs were then calculated using the wet and dry weights measured. Chitosan was extracted from the AIMs with $2 \%$ (v/v) aqueous acetic acid. Two different volume-to-solids ratios (40:1 or 10:1, mL/g dry mass AIMs) and heat treatments (autoclaving at $121^{\circ} \mathrm{C}, 15 \mathrm{psi}$ for $15 \mathrm{~min}$ or refluxing at $95^{\circ} \mathrm{C}$ for $8 \mathrm{~h}$ ) were tested. After the extraction period, the slurries were transferred into tared centrifuge tubes and centrifuged at 3000 RPM for 20 minutes, after which the insoluble fraction was discarded. The $\mathrm{pH}$ of the supernatant was adjusted to $\mathrm{pH} 10$ by the dropwise addition of 4 $\mathrm{M} \mathrm{NaOH}$ in order to precipitate the chitosan out of solution. The suspension was then centrifuged at $3000 \mathrm{RPM}$ for 20 minutes, after which the supernatant was discarded. The chitosan pellets were then washed successively with DI water, $95 \%$ ethanol, and acetone; frozen at $-20^{\circ} \mathrm{C}$ overnight; freeze-dried; and weighed to calculate the chitosan yield ( $\% \mathrm{w} / \mathrm{w}$ dry mass of initial soymeal substrate).

\subsection{Characterization of Fungal Chitosan by FTIR Spectroscopy}

The Fourier transfer infrared (FTIR) spectra of the product fungal chitosan from soymeal fermentation were obtained by analyzing solid powder samples with an Alpha-E FTIR spectrometer (Bruker Optics Inc., Billerica, MA, USA). Sixty-four scans were conducted per sample between wavelength number of $4000-700 \mathrm{~cm}^{-1}$ at a resolution of $2 \mathrm{~cm}^{-1}$. The degree of deacetylation (DDA) of the chitosan samples was estimated according to the method of [24]. The absorbance peak at wavelength number $\approx 1560 \mathrm{~cm}^{-1}$ (amide II) was used as the characteristic signal while the peak at wavelength number $\approx 1070 \mathrm{~cm}^{-1}(\mathrm{C}-\mathrm{O}$ bond stretching) was used as the reference signal. DDA values were calculated using the absorbance ratio $\mathrm{A}_{1560} / \mathrm{A}_{1070}$ and the corresponding calibration curve relating the absorbance ratio to DDA.

\section{Results and Discussion}

\subsection{Characterization of Soybean Processing Residues}

Following analysis, the soybean meals were found to contain almost $50 \%$ by weight proteins, which is roughly five times that of the soy hulls. In terms of the carbohydrate content, soy hulls had around $79 \%$ by weight of carbohydrates, which is almost twice that of the carbohydrates in soymeal. Both meal and hulls contain similar residual fats while the meal has slightly higher moisture and ash contents than the hulls. The high carbohydrates and protein content of these residues could serve as carbon and nitrogen sources for direct fungal growth under solid-state cultivation and support the rationale behind the selection of these agricultural residues as fermentation substrates for chitosan production.

\subsection{Effect of Substrate Type}

Pure soybean meal, soy hulls, and 1:1 meal-hull mixtures were tested as SSF substrates for growth and chitosan production by $M$. rouxii ATCC 24905. The results (Figure 1) show that fungal growth (as measured by glucosamine in the culture) under solid-state cultivation conditions in a pure soymeal culture or in a 1:1 meal/hull mixture were not significantly different, while growth in pure soy hull was the lowest. This result was expected as the substrates containing the soymeal contained higher protein (nitrogen) contents than pure soy hulls, resulting in the increased biosynthesis of fungal mycelial materials. On the other hand, chitosan yield was highest from the pure soymeal cultures $(1.63 \% \pm 0.16 \% \mathrm{w} / \mathrm{w}$ dry substrate) while those from the pure soy hull $(1.26 \% \pm$ $0.15 \%)$ and $1: 1 \mathrm{meal} /$ hull mixtures $(0.97 \pm 0.15)$ were not significantly different. The chitosan yield displayed a 


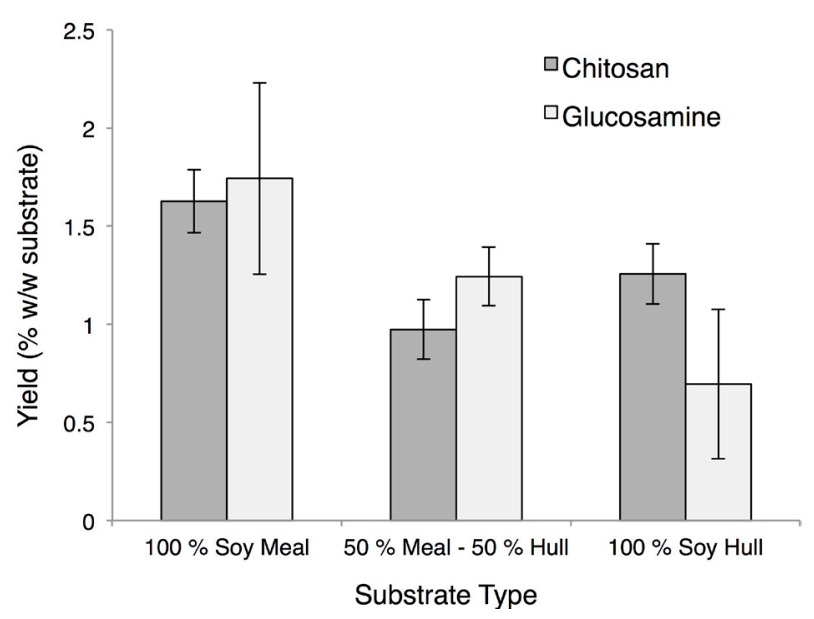

Figure 1. Growth and chitosan production by Mucor rouxii ATCC 24905 on different soybean processing residues. Incubation temperature $-30^{\circ} \mathrm{C}$, initial $\mathrm{pH}$ unadjusted, initial moisture content $-50 \%(\mathrm{w} / \mathrm{w})$, and cultivation time- six days.

positive correlation with fungal growth levels for both the pure soymeal and the 1:1 meal:hull mixtures. Being a fungal cell wall component, the production of chitosan is considered to be growth-associated (i.e., production rates correlate with growth rate) under carbon- and nutrient-rich condition. However, under a condition of high carbohydrate-to-protein proportion such as in soy hull cultures, the chitosan yield displayed an opposite trend relative to fungal growth between the pure soy hull and 1:1 meal/hull cultures. It appears that despite the lower fungal growth level in the soy hull culture, the fungal cell wall mass generated contained a relatively higher composition of chitosan. Based on these results, pure soymeal substrates were utilized in the succeeding fermentation tests.

\subsection{Effect of Incubation Period}

The effect of the length of the cultivation period on fungal growth and chitosan yield using pure soymeal SSF cultures is shown in Figure 2. All other fermentation variables were kept constant at the following levels: $50 \%$ $(\mathrm{w} / \mathrm{w})$ initial substrate moisture content, $30^{\circ} \mathrm{C}$ incubation temperature, and unadjusted initial substrate $\mathrm{pH}$. The fungal growth pattern (as glucosamine) showed a possible diauxic pattern, with a first stationary period attained between 6 to 12 days of cultivation followed by a sharp increase at day 15. It is possible that during the first stationary period, the fungal cells utilized the readily available adhered carbon and nutrients in the soymeal particle surfaces for partial growth and assembly of hydrolytic enzymes (cellulase, proteases, etc.). During the stationary period, the synthesized enzymes were utilized by the fungal cells for the degradation of the polymeric carbohydrates and proteins into more readily assimilable carbon and nutrients, which were then made available for a succeeding exponential growth phase. Despite the observed increase in growth, the results show that maximum chitosan yield was achieved after only six days of cultivation after which chitosan yields fluctuated (not significantly different). Previous studies have indicated that during the attainment of the stationary growth phase, the fungal cell walls may have begun to lose their fluidity and potential for chitosan formation due to consolidation by chitin crystallization and bond formation with other cell wall components, which could lead to reduced extraction yields [22] [25]. Thus, six days was considered to be the optimum length of cultivation period for maximum chitosan yield and was maintained in the succeeding experiments.

\subsection{Effect of Initial Moisture Content}

Figure 3 shows the effect of the initial substrate moisture content on growth and chitosan yield of $M$. rouxii ATCC 24905 in SSF soymeal cultures. In these experiments, the incubation period was set at six days based on the optimum level obtained in the prior experiments, the incubation temperature at $30^{\circ} \mathrm{C}$, and initial substrate $\mathrm{pH}$ was not adjusted. The lowest moisture level tested ( $30 \%$ by wt.) resulted in the lowest fungal biomass growth and chitosan production. However, fungal growth and chitosan yields were not significantly different between 


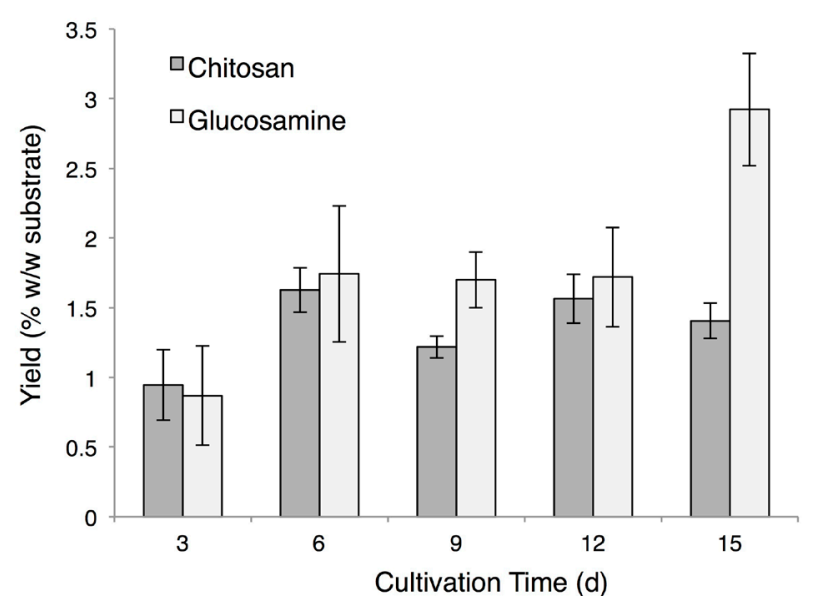

Figure 2. Effect of cultivation time on growth and chitosan yield by Mucor rouxii ATCC 24905 on pure soymeal substrate. Incubation temperature $-30^{\circ} \mathrm{C}$, initial $\mathrm{pH}$ unadjusted, initial moisture content- $50 \%(\mathrm{w} / \mathrm{w})$, and cultivation time - six days.

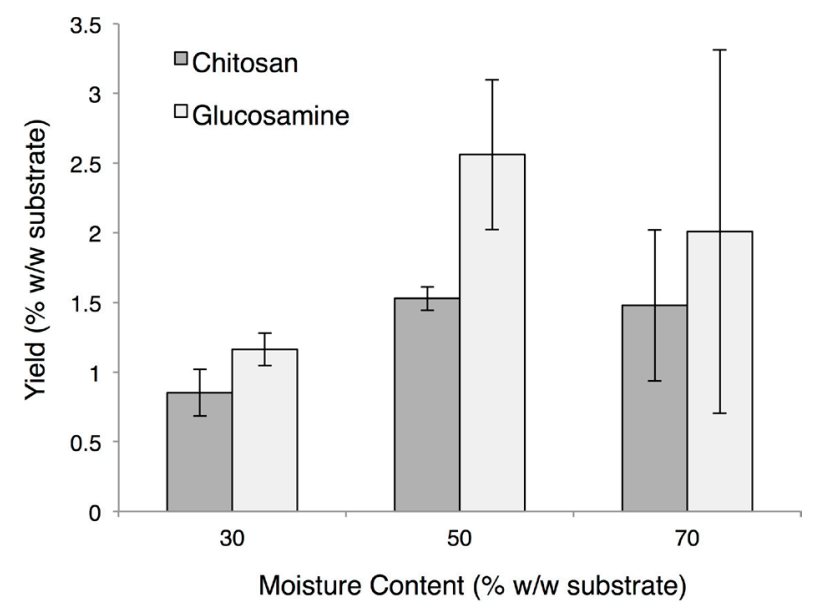

Figure 3. Effect of initial substrate moisture content on growth and chitosan production by Mucor rouxii ATCC 24905 on pure soymeal substrate. Incubation temperature $-30^{\circ} \mathrm{C}$, initial $\mathrm{pH}$ unadjusted, and cultivation time- six days.

the $50 \%$ and $70 \%$ initial moisture content treatments (chitosan yield is between $1 \%$ and $2 \% \mathrm{w} / \mathrm{w}$ dry substrate). The moisture content of the substrate is a critical parameter related to the water activity of the microorganisms in SSF processes where moisture levels are relatively low. The fungal cells need an appropriate level of water in which to exercise the various biochemical reactions for the enzymatic breakdown and uptake of the biomass substrate. The results in this study indicated that M. rouxii ATCC 24905 was able to tolerate a lower substrate moisture content of $50 \%$ for growth and chitosan production in SS unlike other fungal strains previously studied for chitosan production via SSF, that were grown in $60 \%-70 \%$ moisture substrates [12] [22] [26]. Using less water to moisten the soybean meal substrate could mean less dilution of nutrients and growth factors due to water addition in the culture. Furthermore, using soymeal cultures with $70 \%$ initial moisture content appeared to produce high variability in the results. Thus, the initial soymeal substrate moisture content of $50 \%$ was considered to be the optimum level for maximizing fungal growth and chitosan production using the strain M. rouxii ATCC 24905.

\subsection{Effect of Incubation Temperature}

Figure 4 shows the effect of incubation temperature on fungal growth and chitosan production in solid-state 


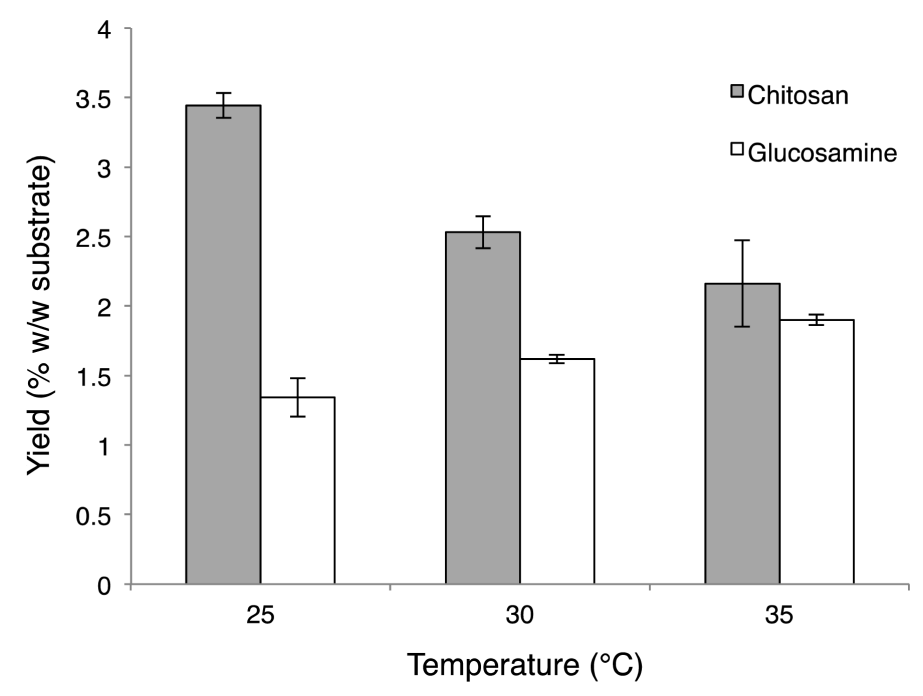

Figure 4. Effect of incubation temperature on growth and chitosan production by Mucor rouxii ATCC 24905 on pure soymeal substrate. Initial $\mathrm{pH}$ unadjusted, initial moisture content- $50 \%(\mathrm{w} / \mathrm{w})$, and cultivation time-six days.

cultures of M. rouxii ATCC 24905. In these experiments, the incubation period was set at six days and the initial moisture content of the soymeal substrate was $50 \%(\mathrm{w} / \mathrm{w})$ based on the optimum levels obtained in the prior experiments. The initial substrate $\mathrm{pH}$ was not adjusted. The experiments demonstrated opposite trends of growth and chitosan yield with increasing incubation temperature. The highest chitosan yield of around $3.44 \% \pm 0.09 \%$ (w/w of total solid substrate) was achieved at $25^{\circ} \mathrm{C}$ and decreased considerably at incubation temperatures of 30 and $35^{\circ} \mathrm{C}$. On the other hand, total fungal growth was shown to increase as the incubation temperature increased. A higher incubation temperature appeared to favor fungal growth in the solid soymeal culture but results in lower chitosan yields. A possible explanation is that the fungal cell walls might be losing their fluidity in favor of a denser and more consolidated structure dominated by chitin, which results in reduction of chitosan composition and/or extractability at incubation temperatures higher than $25^{\circ} \mathrm{C}$. This could be a defense mechanism by the fungal cells to counteract the potentially detrimental effects of high temperatures on fungal growth and metabolism. On the operational standpoint, incubation at lower temperatures could lead to lower costs attributed to heating and humidification of fermentation chambers but could imply the necessary fermentation bed cooling mechanisms to counteract the buildup of temperature gradients across the substrate bed thickness.

\subsection{Effect of Initial Substrate pH}

Figure 5 shows the effect of the initial $\mathrm{pH}$ of the moistened soybean meal substrate on fungal growth and chitosan production in solid-state cultures of $M$. rouxii ATCC 24905. In these experiments, the incubation period was set at six days, the incubation temperature at $30^{\circ} \mathrm{C}$, and initial substrate moisture content was at $50 \%(\mathrm{w} / \mathrm{w})$ according to the results of the prior experiments. The results obtained demonstrate that there are no significant differences in terms of fungal growth and chitosan yields among the initial substrate $\mathrm{pH}$ levels investigated. It seems logical to select the unadjusted $\mathrm{pH}$ as the best condition from the operational cost standpoint, which would not require prior treatment of the soymeal substrate with acid and/or alkali for $\mathrm{pH}$ adjustment. However, an initial pH setting between 5 and 6 might be ideal for helping to minimize the variability of the product chitosan yields and characteristics. Additional tests will be conducted to confirm this hypothesis.

\subsection{Effect of Extraction Conditions}

In addition to testing the effect of the fermentation variables on fungal growth and chitosan yield, different extraction methods were also tested to determine their effects on chitosan recoveries. Different methods were selected from literature. The results of these tests are summarized in Figure 6. In these experiments, the fermentation conditions were kept constant at six days cultivation period, 50\% initial substrate moisture content, unad- 


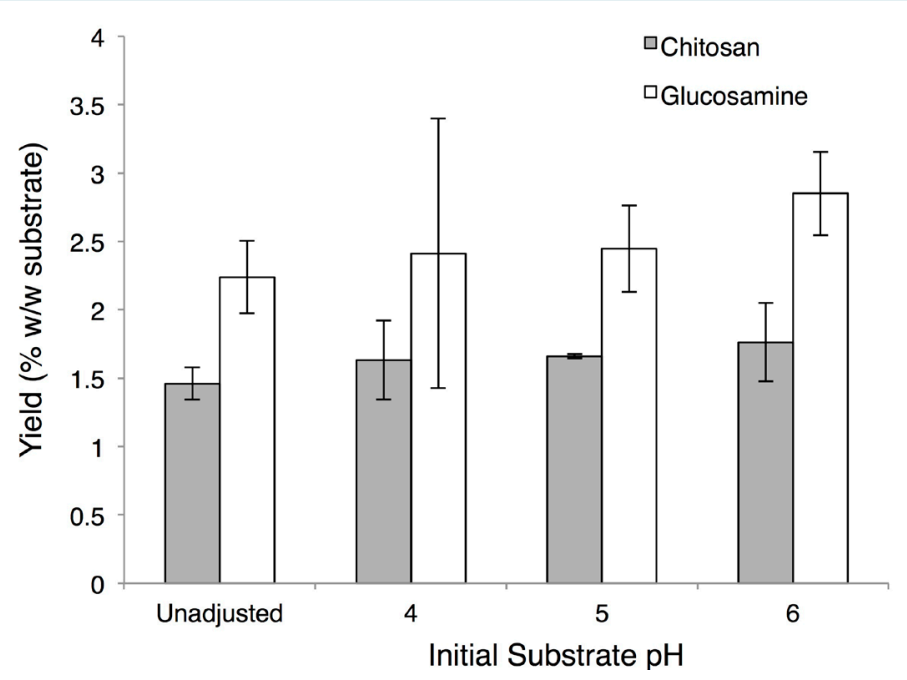

Figure 5. Effect of initial substrate $\mathrm{pH}$ on growth and chitosan production by Mucor rouxii ATCC 24905 on pure soymeal substrate. Incubation temperature $-30^{\circ} \mathrm{C}$, initial moisture content $-50 \%(\mathrm{w} / \mathrm{w})$, and cultivation time - six days.

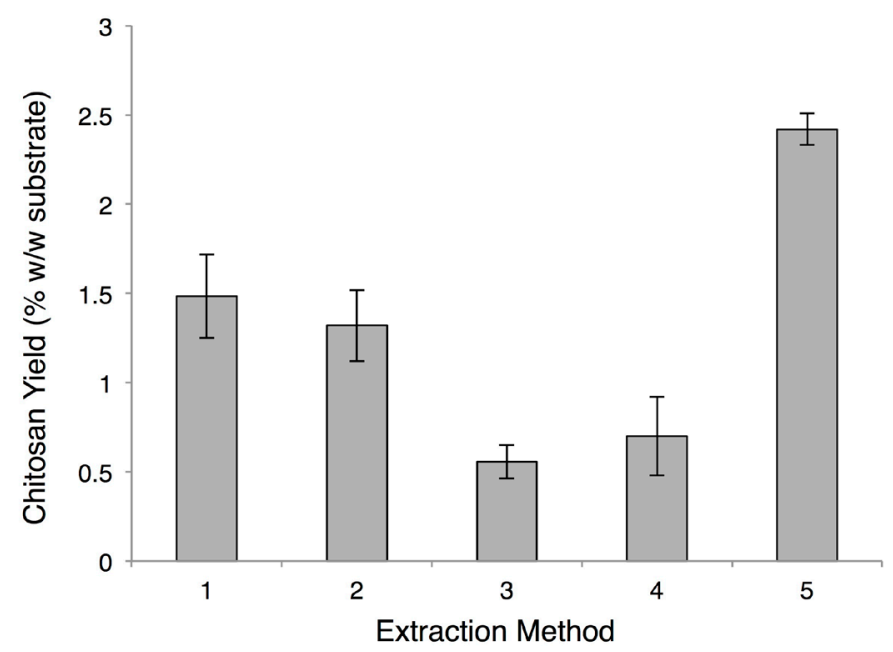

Figure 6. Effect of extraction method on growth and chitosan yield by Mucor rouxii ATCC 24905 . Method $1-1 \mathrm{M} \mathrm{NaOH}$ at $121^{\circ} \mathrm{C}$ for $20 \mathrm{~min}$ (30 mL per g substrate) followed by $2 \%(\mathrm{v} / \mathrm{v})$ acetic acid $(40 \mathrm{~mL}$ per $\mathrm{g}$ substrate) at $95^{\circ} \mathrm{C}$ with refluxing for $8 \mathrm{~h}$. Method $2-1 \mathrm{M} \mathrm{NaOH}$ at $121^{\circ} \mathrm{C}$ for $20 \mathrm{~min}(10 \mathrm{~mL}$ per $\mathrm{g}$ substrate) followed by $2 \%(\mathrm{v} / \mathrm{v})$ acetic acid $(10$ $\mathrm{mL}$ per $\mathrm{g}$ substrate) at $95^{\circ} \mathrm{C}$ with refluxing for $8 \mathrm{~h}$. Method $3-46 \%$ (w/w) $\mathrm{NaOH}$ at $46^{\circ} \mathrm{C}$ for $13 \mathrm{~h}(30 \mathrm{~mL}$ per g substrate) followed by $2 \%$ $(\mathrm{v} / \mathrm{v})$ acetic acid $\left(40 \mathrm{~mL}\right.$ per $\mathrm{g}$ substrate) at $95^{\circ} \mathrm{C}$ with refluxing for $8 \mathrm{~h}$. Method $4-46 \%(\mathrm{w} / \mathrm{w}) \mathrm{NaOH}$ at $46^{\circ} \mathrm{C}$ for $13 \mathrm{~h}(10 \mathrm{~mL}$ per g substrate) followed by $2 \%(\mathrm{v} / \mathrm{v})$ acetic acid $\left(10 \mathrm{~mL}\right.$ per $\mathrm{g}$ substrate) at $95^{\circ} \mathrm{C}$ with refluxing for $8 \mathrm{~h}$. Method $5-1 \mathrm{M} \mathrm{NaOH}$ at $121^{\circ} \mathrm{C}$ for $20 \mathrm{~min}(30 \mathrm{~mL}$ per $\mathrm{g}$ substrate) followed by $2 \%(\mathrm{v} / \mathrm{v})$ acetic acid $(40 \mathrm{~mL}$ per $\mathrm{g}$ substrate) at $121^{\circ} \mathrm{C}$ for $20 \mathrm{~min}$.

justed initial substrate $\mathrm{pH}$, and $30^{\circ} \mathrm{C}$ incubation temperature. Figure 6 shows that chitosan recoveries differed significantly among the different methods investigated with regards to the concentration of reagents used as well as the severity of the heat treatment method used. For instance, Methods 1 and 2 utilized the same reagent concentrations but with differing volume ratios, while Method 2 used a lower volume of reagent. Although a higher volume-to-mass ratio for both the base and acid reagents (Method 1) resulted in a slightly higher chitosan re- 
covery, the results were not significantly different. The same case was observed with Methods 3 and 4, which used a higher base concentration but with heating and refluxing instead of autoclaving. Compared with Methods 1 and 2, Methods 3 and 4 resulted in a significantly lower chitosan recovery. This finding implies the importance of the base-assisted deproteinization step for maximizing the chitosan yield. However, Figure $\mathbf{5}$ also shows that when a more severe reaction condition (i.e., autoclaving) was employed for the acetic acid extraction of chitosan step, the chitosan recovery was significantly higher than all the other extraction methods tested. Despite this, a more thorough economic and cost-benefit study should be made to justify the use of more severe extraction conditions and correlations must be made with the effect of fermentation conditions.

\subsection{Characterization of Fungal Chitosan Using FTIR}

The chitosan extracted from Mucor rouxii ATCC 24905 fungal cultures in soybean meal was characterized by FTIR spectroscopy and compared with commercially available chitosan. The purpose of this analysis was to confirm the identity of the extracted chitosan pellets and to estimate the degree of deacetylation (DDA) of the fungal chitosan product, an important parameter that influences the activity of the chitosan polymer for its intended applications. The DDA of the fungal chitosan produced from solid-state fermentation of soybean meal was estimated using the absorbances of the FTIR signals at approximate wavelength numbers of 1560 and 1070 $\mathrm{cm}^{-1}$ (Shigemasa et al., 1996) As shown in Figure 7, the signal at $1560 \mathrm{~cm}^{-1}$ corresponds to the characteristic band of the amide group (Amide II) in the N-acetylglucosamine residue of chitin, which accounts for more than $90 \%$ of the monomer residues in the polymer chain. Although definitions vary among different sources, the chitin chain is identified as chitosan if the polymer chain contains a significant proportion of the deacetylated glucosamine residue. Thus the intensity of the band at $1560 \mathrm{~cm}^{-1}$ is expected to decrease as the DDA increases. The signal at $1070 \mathrm{~cm}^{-1}$ corresponding to $\mathrm{C}-\mathrm{O}$ bond stretching in the chitin/chitosan monomers was used as the reference band. Using the calibration curve developed by Shigemasa et al., the DDA of representative samples of fungal chitosan from different fermentation and extraction conditions was estimated to be between $55 \%-60 \%$.

\section{Conclusion}

Solid-state fermentation (SSF) of soybean processing residues using the fungus Mucor rouxii ATCC 24905 for chitosan production was for the first time investigated. The optimum SSF conditions for maximum chitosan

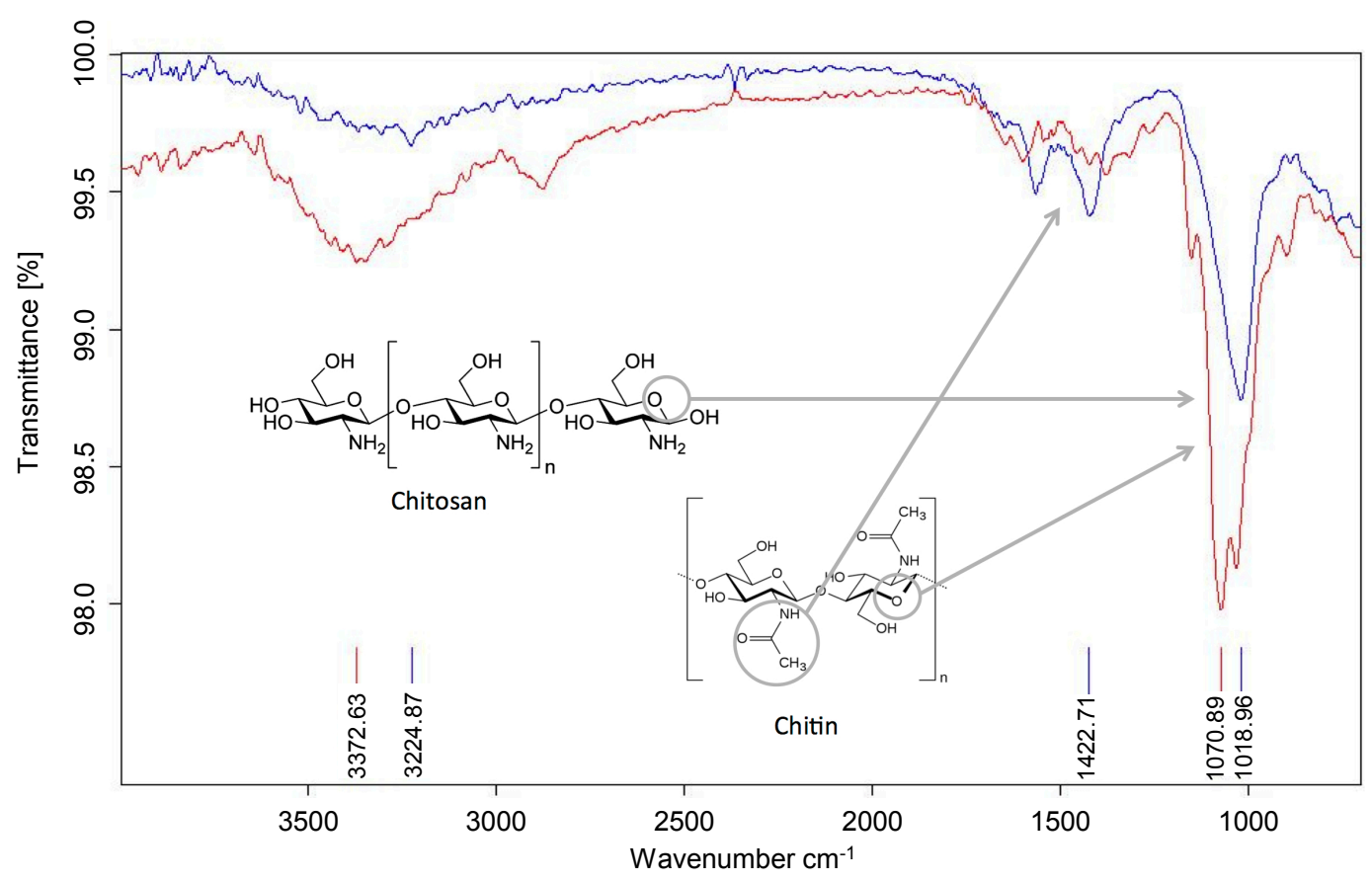

Figure 7. FTIR spectra of commercial chitosan (red) and Mucor rouxii ATCC 24905 chitosan from fermentationofpure soymeal substrate (blue). 
yields were determined. FTIR analysis of the fungal chitosan derived from soybean meal fermentation confirmed its molecular identity and estimated its degree of deacetylation to be around 55\% - 60\%. Extraction conditions such as the concentration of acid and base reagents and severity of heat treatment were also shown to influence the chitosan yield.

\section{Acknowledgements}

The authors would like to acknowledge the United Soybean Board (USB) for providing the funding for this research (USB Project \# 1440-512-5283): Archer Daniels Midland, Zeeland Farms, and CHS for supplying the soybean meal and hull samples; and Dr. Linda Kim-Habermehl of USB/Omni Tech International, Ltd. for her valuable inputs on the project.

\section{References}

[1] Castro, S.P.M. and Paulín, E.G.L. (2012) Is Chitosan a New Panacea? Areas of Application. In: Karunaratne, D.N., Ed., The Complex World of Polysaccharides, ISBN: 978-953-51-0819-1, InTech. http://www.intechopen.com/books/the-complex-world-of-polysaccharides/is-chitosan-a-new-panacea-areas-of-applicat $\underline{\text { ion }}$

[2] Kaur, S., and Dhillon, G.S. (2014) The Versatile Biopolymer Chitosan: Potential Sources, Evaluation of Extraction Methods and Applications. Critical Reviews in Microbiology, 2, 155-175. http://dx.doi.org/10.3109/1040841X.2013.770385

[3] Badawy, M.E.I. and Rabea, E.I. (2011) A Biopolymer Chitosan and Its Derivatives as Promising Antimicrobial Agents against Plant Pathogens and Their Applications in Crop Protection. International Journal of Carbohydrate Chemistry, 2011, Article ID: 460381, 29 p. http://dx.doi.org/10.1155/2011/460381

[4] Dutta, P.K., Dutta, J., and Tripathi, V.S. (2004) Chitin and Chitosan : Chemistry, Properties and Applications. Journal of Scientific \& Industrial Research, 63, 20-31. http://nopr.niscair.res.in/handle/123456789/5397

[5] Bruck, W.M., Slater, J.W., and Carney, B.F. (2011) Chitin and Chitosan from Marine Organisms. In: Kim, S.K., Ed., Chitin, Chitosan, Oligosaccharides, and Their Derivatives: Biological Activities and Applications, CRC Press, Boca Raton, 11-24.

[6] Knezevic-Jugovic, Z., Petronijevic, Z., and Smelcerovic, A. (2011) Chitin and Chitosan from Microorganisms. In: Kim, S.K., Ed., Chitin, Chitosan, Oligosaccharides, and Their Derivatives: Biological Activities and Applications, CRC Press, Boca Raton, 25-36.

[7] Peter, M.G. (2002) Chitin and Chitosan in Fungi. Biopolymers Online, 6, 123-157. http://dx.doi.org/10.1002/3527600035.bpol6005

[8] White, S.A., Farina, P.R. and Fulton, I. (1979) Production and Isolation of Chitosan from Mucor rouxii. Applied and Environmental Microbiology, 38, 323-328.

[9] Tan, S.C., Tan, T.K., Wong, S.M. and Khorb, E. (1996) The Chitosan Yield of Zygomycetes at Their Optimum Harvesting Time. Carbohydrate Polymers, 30, 239-242. http://dx.doi.org/10.1016/S0144-8617(96)00052-5

[10] Pochanavanich, P. and Suntornsuk, W. (2002) Fungal Chitosan Production and Its Characterization. Letters in Applied Microbiology, 35, 17-21. http://dx.doi.org/10.1046/j.1472-765X.2002.01118.x

[11] McGahren, W.J., Perkinson, G.A., Growich, J.A., Leese, R.A. and Ellestad, G.A. (1984) Chitosan by Fermentation. Process Biochemistry, 19, 88-90.

[12] Kleekayai, T. and Suntornsuk, W. (2010) Production and Characterization of Chitosan Obtained from Rhizopus oryzae Grown on Potato Chip Processing Waste. World Journal of Microbiology and Biotechnology, 27, 1145-1154. http://dx.doi.org/10.1007/s11274-010-0561-X

[13] Wang, W., Du, Y., Qiu, Y., Wang, X., Hu, Y., Yang, J., Cai, J. and Kennedy, J.F. (2008) A New Green Technology for Direct Production of Low Molecular Weight Chitosan. Carbohydrate Polymers, 74, 127-132. http://dx.doi.org/10.1016/j.carbpol.2008.01.025

[14] Nwe, N. and Stevens, W.F. (2002) Production of Fungal Chitosan by Solid Substrate Fermentation Followed by Enzymatic Extraction. Biotechnology Letters, 24, 131-134. http://dx.doi.org/10.1023/A:1013850621734

[15] Suntornsuk, W., Pochanavanich, P. and Suntornsuk, L. (2002) Fungal Chitosan Production on Food Processing ByProducts. Process Biochemistry, 37, 727-729. http://dx.doi.org/10.1016/S0032-9592(01)00265-5

[16] Bartnicki-Garcia, S. and Nickerson, W.J. (1962) Isolation, Composition, and Structure of Cell Walls of Filamentous and Yeast-Like Forms of Mucor rouxii. Biochimica et Biophysica Acta, 58, 102-119. http://dx.doi.org/10.1016/0006-3002(62)90822-3 
[17] Chatterjee, S., Adhya, M., Guha, A.K. and Chatterjee, B.P. (2005) Chitosan from Mucor rouxii: Production and Physico-Chemical Characterization. Process Biochemistry, 40, 395-400. http://dx.doi.org/10.1016/j.procbio.2004.01.025

[18] American Soybean Association (2014) SoyStats 2014. Soybean Facts. http://soystats.com/facts/

[19] American Soybean Association (2014) SoyStats 2014. US Yield and Production: Production History. http://soystats.com/u-s-yield-production-production-distory/

[20] Zhang, L.Y., Li, D.F. and Qiao, S.Y. (2003) Effects of Stachyose on Performance, Diarrhea Incidence and Intestinal Bacteria in Weanling Pigs. Archives of Animal Nutrition, 57, 1-10. http://dx.doi.org/10.1080/0003942031000086662

[21] Roopesh, K., Ramachandran, S., Nampoothiri, K.M., Szakacs, G. and Pandey, A. (2006) Comparison of Phytase Production on Wheat Bran and Oilcakes in Solid-State Fermentation by Mucor racemosus. Bioresource Technology, 97, 506-511. http://dx.doi.org/10.1016/j.biortech.2005.02.046

[22] Crestini, C., Kovac, B. and Giovannozzi-Sermanni, G. (1996) Production and Isolation of Chitosan by Submerged and Solid-State Fermentation from Lentinus edodes. Biotechnology and Bioengineering, 50, 207-210. http://dx.doi.org/10.1002/bit.260500202

[23] Rane, K.D. and Hoover, D.G. (1993) Production of Chitosan by Fungi. Food Biotechnology, 7, 11-33. http://dx.doi.org/10.1080/08905439309549843

[24] Shigemasa, Y., Matsuura, H., Sashiwa, H. and Saimoto, H. (1996) Evaluation of Different Absorbance Ratios from Infrared Spectroscopy for Analyzing the Degree of Deacetylation in Chitin. International Journal of Biological Macromolecules, 18, 237-242. http://dx.doi.org/10.1016/0141-8130(95)0179-3

[25] Nwe, N., Furuike, T. and Tamura, H. (2010) Production of Fungal Chitosan by Enzymatic Method and Applications in Plant Tissue Culture and Tissue Engineering: 11 Years of Our Progress, Present Situation and Future Prospects. In: Elnashar, M., Ed., Biopolymers, ISBN: 978-953-307-109-1, InTech. http://dx.doi.org/10.5772/10261 http://www.intechopen.com/books/biopolymers/production-of-fungal-chitosan-by-enzymatic-method-and-applicationsin-plant-tissue-culture-and-tissu

[26] Khalaf, S.A. (2004) Production and Characterization of Fungal Chitosan under Solid-State Fermentation Conditions. International Journal of Agriculture \& Biology, 6, 1033-1036.

http://www.fspublishers.org/published_papers/4088_..pdf 
Scientific Research Publishing (SCIRP) is one of the largest Open Access journal publishers. It is currently publishing more than 200 open access, online, peer-reviewed journals covering a wide range of academic disciplines. SCIRP serves the worldwide academic communities and contributes to the progress and application of science with its publication.

Other selected journals from SCIRP are listed as below. Submit your manuscript to us via either submit@scirp.org or Online Submission Portal.
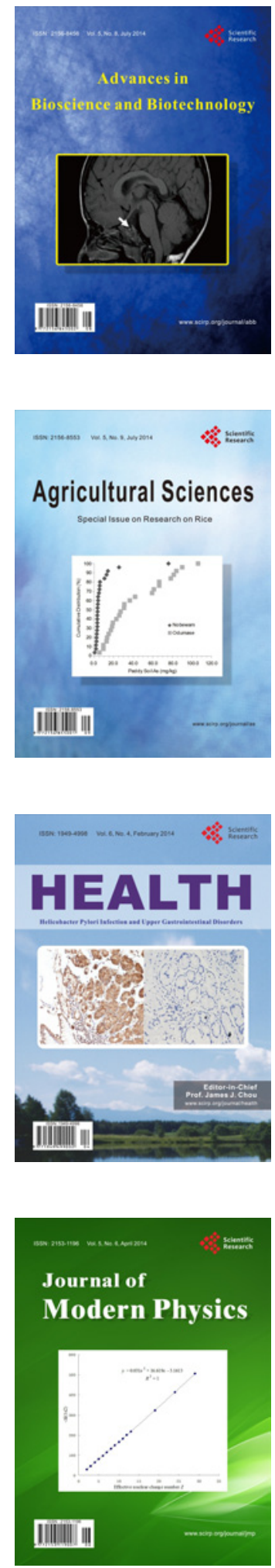
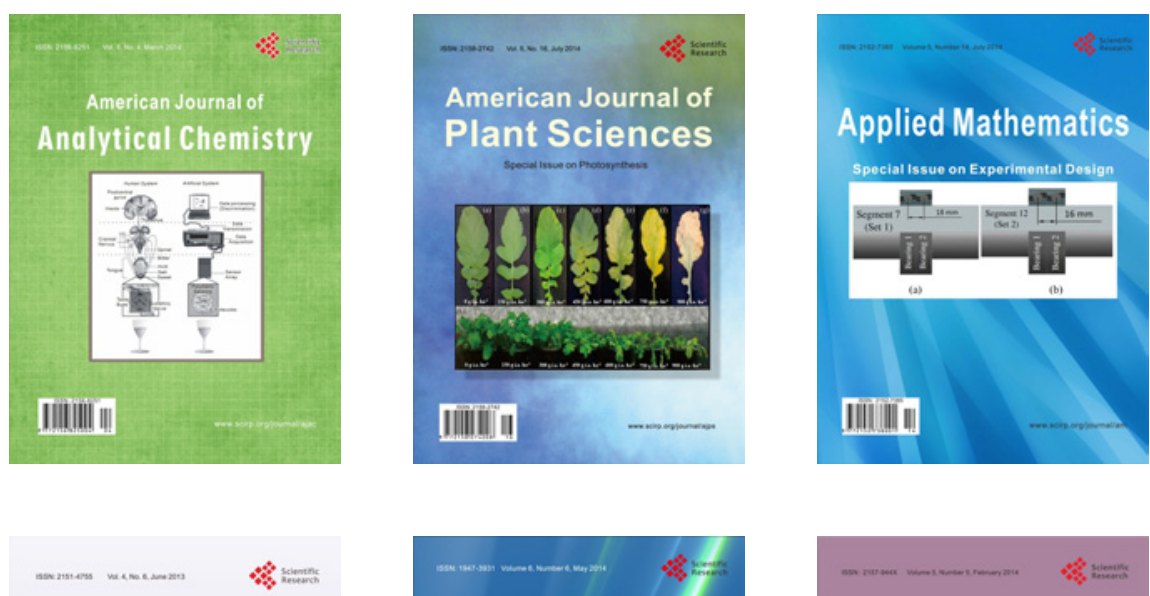

Creative Education
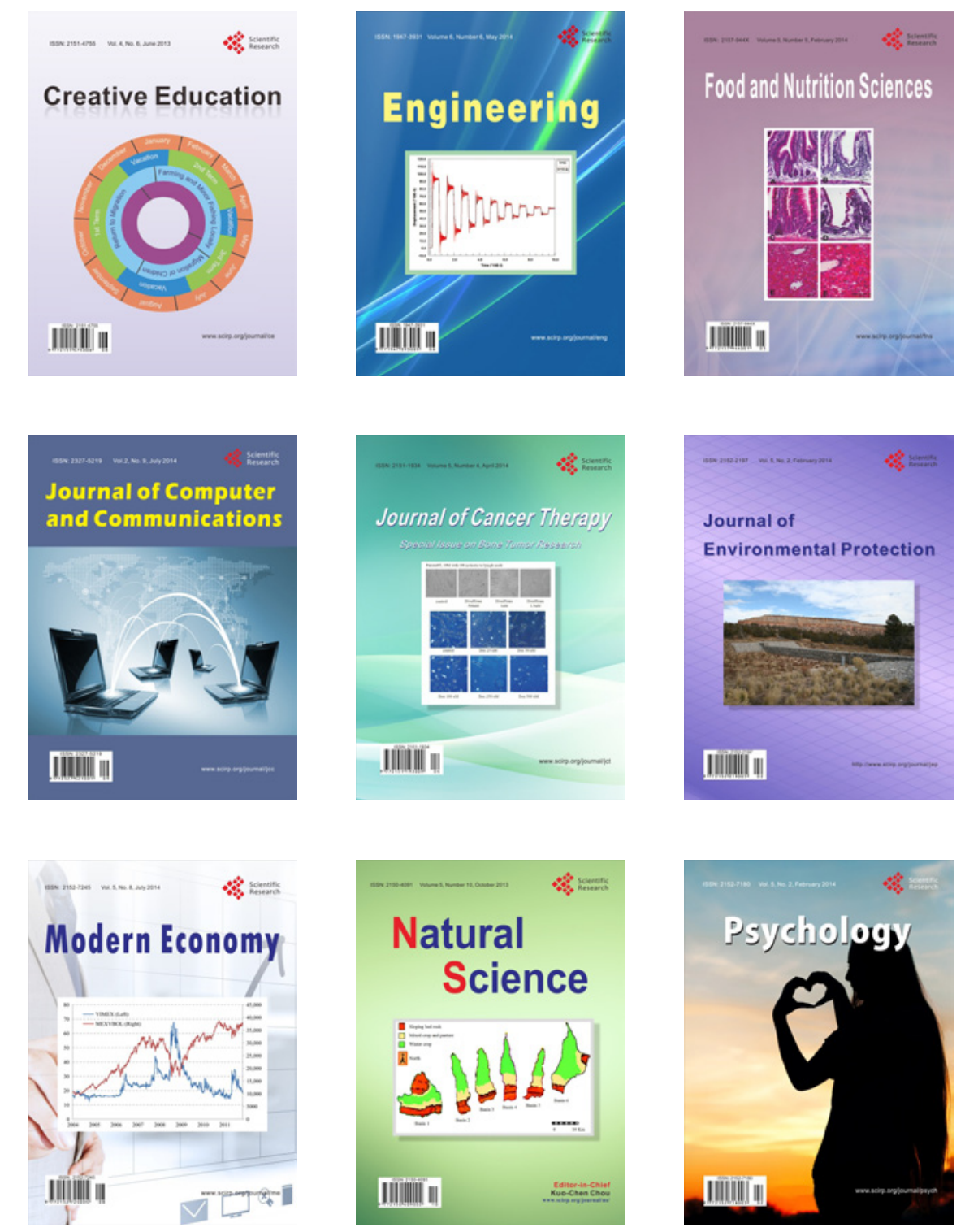